\title{
Rhinosinusitis without nasal polyps in COPD
}

\author{
Marte Rystad Øie (10 ${ }^{1,2}$, Sarah Bettina Dahlslett ${ }^{1,2}$, Malcolm Sue-Chu ${ }^{3,4}$, \\ Anne-S. Helvik ${ }^{1,5}$, Sverre Karmhus Steinsvåg ${ }^{6,7}$ and \\ Wenche Moe Thorstensen ${ }^{1,2}$
}

Affiliations: ${ }^{1}$ Dept of Otolaryngology, Head and Neck Surgery, St. Olavs University Hospital, Trondheim, Norway. ${ }^{2}$ Dept of Neuromedicine and Movement Science, Norwegian University of Science and Technology (NTNU), Trondheim, Norway. ${ }^{3}$ Dept of Circulation and Medical Imaging, Norwegian University of Science and Technology, Trondheim, Norway. ${ }^{4}$ Dept of Thoracic Medicine, St. Olavs University Hospital, Trondheim, Norway. ${ }^{5}$ Dept of Public Health and Nursing, Norwegian University of Science and Technology, Trondheim, Norway. ${ }^{6}$ Dept of Otolaryngology, Head and Neck Surgery, Sørlandet Hospital, Kristiansand, Norway. ${ }^{7}$ Haukeland University Hospital, Bergen, Norway.

Correspondence: Marte Rystad Øie, Dept of Otolaryngology, Head and Neck Surgery, St. Olavs University Hospital, 3250 Torgarden, 7006 Trondheim, Norway. E-mail: marte.oiedgmail.com

ABSTRACT The validity of the united airway disease concept for rhinosinusitis (RS) and chronic obstructive pulmonary disease (COPD) has been questioned because of methodological limitations in previous studies. In this study we investigated the prevalence of RS without nasal polyps (RSsNP) and the severity of sinonasal symptoms in COPD and a corresponding control group. We also evaluated the diagnostic accuracy of these symptoms for RSsNP in COPD. 90 COPD patients and 93 controls were included in an observational cross-sectional study where globally accepted diagnostic criteria of RS and COPD (EPOS 2012 and GOLD) were incorporated; symptomatic and endoscopic criteria for the diagnosis of RS, and spirometry with reversibility for diagnosis of COPD. RS symptoms were identified by responses to the sinonasal outcome test (SNOT-22), nasal endoscopy identified signs of sinonasal disease and discriminated between RS with and without nasal polyps, and visual analogue scales (VAS) rated the severity of sinonasal symptoms. We found RSsNP in 51\% of our COPD patients which is threefold greater than in the control group $(\mathrm{p}<0.001)$. Nasal discharge $(72 \%)$ and nasal obstruction $(62 \%)$ were the two most frequently reported symptoms in COPD. The diagnostic accuracy for RSsNP is better for the composite VAS for rhinological symptoms than for facial symptoms. We conclude that RSsNP is present in $51 \%$ of our COPD patients, which is significantly more prevalent compared to a corresponding control group. These results suggest that COPD is associated with RS.

@ERSpublications

Rhinosinusitis is associated with COPD and is significantly more prevalent $(51 \%)$ compared to controls https://bit.ly/2YrIsmm

Cite this article as: Øie MR, Dahlslett SB, Sue-Chu M, et al. Rhinosinusitis without nasal polyps in COPD. ERJ Open Res 2020; 6: 00015-2020 [https://doi.org/10.1183/23120541.00015-2020].

Received: 13 Jan 2020 | Accepted after revision: 28 April 2020

Copyright $\odot$ ERS 2020. This article is open access and distributed under the terms of the Creative Commons Attribution Non-Commercial Licence 4.0. 


\section{Introduction}

Rhinosinusitis (RS) is a major upper airway disease with an overall prevalence of $10.9 \%$ in Europe, and has a significant socioeconomic impact [1]. The European position paper on rhinosinusitis and nasal polyps (EPOS) defines RS in adults as an inflammation of the nose and paranasal sinuses, and diagnosis requires the presence of symptomatic and either endoscopic or radiologic criteria. The symptomatic criteria are the presence of at least two of the following symptoms: nasal obstruction, nasal discharge (anterior/posterior nasal drip), reduction/loss of smell and facial pain/pressure, whereof one should be nasal obstruction or nasal discharge [2]. Confirmation of sinus disease using endoscopic or radiological criteria is a requirement, as symptoms are nonspecific and can be manifestations of other diseases, such as upper respiratory tract infection, allergic rhinitis and migraine [3]. The nasal endoscopic criteria are the presence in one or both nasal cavities of nasal polyps, and/or mucopurulent discharge and/or oedema or mucosal obstruction primarily in the middle nasal cavity. The radiological criteria are mucosal changes within the ostiomeatal complex or sinuses on computed tomography examination. Of the two clinical phenotypes of RS, without nasal polyps (RSsNP) is twice as prevalent as with nasal polyps (RSwNP) [4].

The united airway disease (UAD) concept is based on epidemiological and clinical studies that have reported an association of sinonasal diseases, such as allergic rhinitis and RS, with obstructive airway diseases. According to this concept, the upper and lower airways should be considered as a single airway, as disease processes in the lower airway such as inflammation, may affect the upper airway and vice versa $[5,6]$. Moreover, optimal management of lower airway disease requires that upper airway disease should be assessed and treated appropriately, as exemplified in asthma by the need to assess for and treat coexisting allergic rhinitis and polyps [7].

Chronic obstructive pulmonary disease (COPD) is an inflammatory, progressive lower airway disease with persistent airflow limitation [8]. The main risk factor is exposure to cigarette smoke in developed countries and to biomass smoke in poorly ventilated homes in developing countries [9]. Several epidemiological and clinical studies have suggested an association of COPD with sinonasal symptoms; $40-88 \%$ of COPD patients report either current, recurrent or permanent symptoms, such as rhinorrhoea/nasal discharge, nasal obstruction and/or sneezing [10-14]. However, in a recent review on sinonasal disease and inflammation in COPD, the validity of this association was questioned as the reviewed studies had methodological limitations, such as the absence of a control population, self-reported COPD diagnosis and use of nonstandardised criteria for the diagnosis of RS and COPD [15].

In this study, we investigated the prevalence of RSsNP in COPD and a corresponding control group with respect to age and sex, applying EPOS 2012 and Global Initiative for Chronic Obstructive Lung Disease (GOLD) criteria for the clinical diagnosis of RS and COPD, respectively. We also investigated the severity of sinonasal symptoms and evaluated the diagnostic accuracy of these symptoms for RS in COPD.

\section{Material and methods}

\section{Study design and subjects}

The study was conducted as an observational cross-sectional study between February 2016 and December 2017 at St. Olavs University Hospital, Trondheim, Norway. COPD patients were recruited from the respiratory outpatient and physical therapy clinics at St. Olavs University Hospital, a private pulmonology practice and several general practitioner offices in Trondheim. Controls were recruited from the general

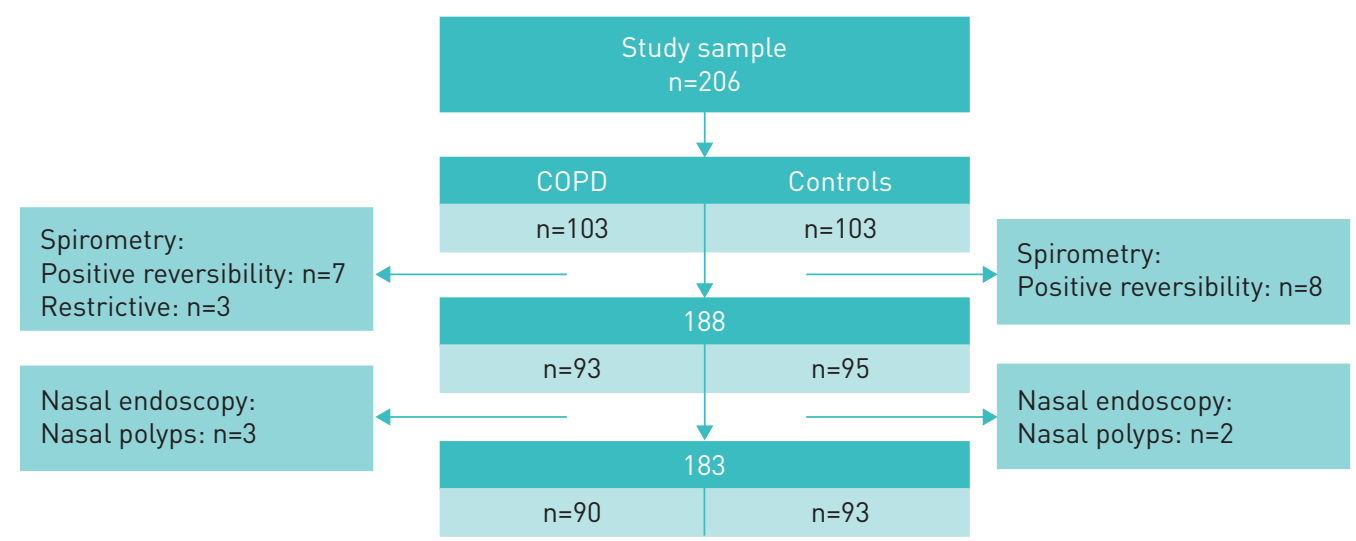

FIGURE 1 Overview of the study sample showing excluded subjects in each group with positive reversibility test and nasal polyps. 
population, via invitation to different workplaces in Trondheim, multiple retirement associations, and posters in various shopping malls and on the hospital's social media page. Inclusion criteria were age 40-80 years and no history of sinonasal surgery in both groups, no known lower airway disease in the control group and a post-bronchodilator forced expiratory volume in $1 \mathrm{~s}\left(\mathrm{FEV}_{1}\right)$ to forced vital capacity (FVC) ratio of $<0.7$ with a negative bronchodilator reversibility test in the COPD group. For both groups, exclusion criteria were any of the following: known underlying systemic diseases that could potentially affect the nose, such as eosinophilic polyangiitis (EGPA) and granulomatous polyangiitis (GPA), cystic fibrosis, primary ciliary dyskinesia/Kartagener syndrome and sarcoidosis, nasal tumours, asthma, current treatment for malignancy, symptoms of common cold within the previous 2 weeks, use of long-term oxygen therapy, a positive bronchodilator reversibility test, presence of nasal polyps on endoscopy, COPD exacerbation within the last 6 weeks. An overview of the study sample is presented in figure 1.

All subjects gave oral and written consent for participation in the study. The study was approved by the National Ethical Committee of Norway (reference number 2015/2017), and investigations were performed in accordance with the principles of the Declaration of Helsinki/Hong Kong.

\section{Methods}

Subjects were instructed to discontinue the use of systemic antihistamine and corticosteroids at 4 days and nasal decongestants at $12 \mathrm{~h}$ prior to the inclusion visit. Nasal corticosteroids were not discontinued. COPD patients were instructed not to take their morning inhaled medication.

Weight and height were recorded for calculation of body mass index (BMI), and spirometry with reversibility test and skin-prick test (SPT) were performed. Subjects completed self-administered questionnaires on smoking habits, symptoms of allergy affecting the airways and sinonasal symptoms. The presence and severity of the latter were evaluated by the sinonasal outcome test-22 (SNOT-22) [16], which is a 22-item questionnaire on nasal and non-nasal symptoms and is a widely recognised and validated tool for RS $[2,16,17]$, and visual analogue scales (VAS), as recommended by EPOS 2012 [2]. An interview and clinical examination with nasal endoscopy were performed by one of three otolaryngologists committed to the study.

\section{Spirometry with reversibility test}

Pulmonary function tests were performed before and after administration of $0.4 \mathrm{mg}$ salbutamol aerosol by spacer and in accordance with European Respiratory Society (ERS) guidelines for spirometry [18]. Predicted reference values of CRAPO et al. [19] were used.

\section{Skin-prick test}

SPT was performed with an allergen panel consisting of birch, grass and mugwort pollen, cladosporium, house dust mite (Dermatophagoides pteronyssinus), and horse, dog and cat epithelia, together with positive and negative controls (Soluprick SQ, ALK-Abello, Horsholm, Denmark). A positive result was defined as a wheal $\geqslant 3 \mathrm{~mm}$ in diameter $15 \mathrm{~min}$ after application [20].

\section{Allergy status}

Allergic rhinitis was diagnosed based on self-reported symptoms characteristic for rhinoconjunctivitis on exposure to the specific allergen(s) found positive on SPT. The self-reported symptoms must have been present within the last 12 months.

\section{Smoking status}

Pack-year exposure was calculated, and subjects were categorised into current, former and never-smokers.

\section{Nasal endoscopy}

Nasal endoscopy was performed with a $2.7 \mathrm{~mm}, 0^{\circ}$ True View II endoscope (Olympus, Japan), and the mucosa in each nasal cavity was evaluated for oedema and mucopurulent discharge. A positive nasal endoscopy was defined as unilateral or bilateral presence of oedema in the middle meatus and/or mucopurulent discharge primarily in the middle nasal cavity [2].

\section{COPD diagnosis confirmation}

The COPD diagnosis was confirmed by the presence of airflow obstruction, defined as an increase of less than $12 \%$ and $200 \mathrm{~mL}$ in $\mathrm{FEV}_{1}$ after administration of salbutamol by inhalation and a post-bronchodilator $\mathrm{FEV}_{1} / \mathrm{FVC}$ ratio of $<0.7$ [8]. Severity of airflow obstruction was graded according to the GOLD 2014 criteria [8]. 
Symptomatic criteria of rhinosinusitis

The symptomatic criteria defined by EPOS were considered present if the respondent had at least two of the four symptoms, of which one was nasal obstruction or nasal discharge. This was determined by dichotomisation of responses to the following items in the SNOT-22 questionnaire: item 4, nasal obstruction; at least one of the following items, nasal discharge (anterior/posterior nasal drip): item 1 (need to blow nose), item 3 (runny nose), item 7 (post-nasal discharge), item 8 (thick nasal discharge); item 5 (loss of taste and smell), reduction/loss of smell; item 12, facial pain/pressure. The response range for each item is $0-5$; with 0 , no problem and 5, problem as bad as it could be. A response of $0-1$ and $2-5$ was defined as "symptom not present" and "symptom present", respectively [21].

\section{Diagnosis of rhinosinusitis without nasal polyps}

The clinical diagnosis of RSsNP was defined as the presence of symptomatic criteria of RS and a positive nasal endoscopy.

Assessment of symptom severity and diagnostic accuracy of sinonasal symptoms

Subscales for rhinological and ear/facial symptoms were constructed by summation of responses to item $1-5,7$ and 8 and item 9-12, respectively, in the SNOT-22 questionnaire [16].

The severity of each of the following symptoms was self-assessed on VAS from 0 to $100 \mathrm{~mm}$, with 0 representing "not troublesome" and 100 "worst thinkable troublesome" [22]: nasal obstruction, nasal discharge (anterior/posterior nasal drip), reduced sense of smell, facial pain, sinus pressure, mouth breathing, sneezing and headache. VAS responses to the first three symptoms were summated to obtain a composite VAS for rhinological symptoms and responses to the following two were summated to obtain a composite VAS for facial symptoms.

The diagnostic accuracy of VAS responses [23] for a diagnosis of RSsNP was evaluated by construction of receiver operating characteristics (ROC) curves of the composite VAS for rhinological and facial symptoms for both groups.

\section{Statistical analysis}

SPSS version 25 (Statistical Packages for the Social Sciences, for Windows, USA) was used for statistical analysis. Continuous variables with a positively skewed distribution were rescaled and $\log _{10}$ transformed to obtain a normal distribution prior to analysis. Comparative analyses between groups were performed with t-tests for continuous variables and the Chi-squared statistic, with Fisher's exact test when appropriate, for categorical variables. Group comparisons with adjustments for age and sex were also performed using linear regression for continuous variables and logistic regression for categorical variables. The results of the regression analyses did not deviate from the results of the Chi-squared and t-tests, thus results are presented as mean \pm SD and frequencies and percentages, respectively. Area under the ROC curve with $95 \%$ confidence intervals were computed for composite VAS for rhinological and facial symptoms to compare the diagnostic accuracy, and a value $>0.8$ has a good diagnostic accuracy [24]. Independent group area difference under the ROC curve was used for tests of statistical significance between COPD and control groups. The level of significance was set at the standard two-sided level of $5 \%$.

\section{Results}

Subject characteristics

In the studied sample, the mean age of 90 subjects with COPD and 93 control subjects were 66.2 and 63.7 years, respectively. Sex and BMI were evenly distributed in the two groups. The prevalence of allergic rhinitis was almost three times higher in control subjects than in COPD subjects (table 1). Airflow limitation was moderate (GOLD2) in 44\% and severe (GOLD3) in 37\% of COPD subjects (table 2).

\section{Symptoms and diagnostic criteria of RS}

The prevalence of three of the four symptoms of RS were significantly greater in the COPD group than in the control group (figure 2a). In both groups, nasal discharge was the most prevalent, followed in decreasing order by nasal obstruction, impaired taste and smell and facial pain/pressure.

The prevalence of symptomatic criteria for RS in COPD subjects was twice the prevalence in controls, (57 $(63 \%)$ versus $28(30 \%), \mathrm{p}<0.001)$ and the endoscopic criteria was 1.5 -fold more prevalent in COPD than in controls (63 (70\%) versus $42(45 \%), \mathrm{p}=0.003)$ (figure 3 ).

\section{Prevalence of RSSNP}

The prevalence of RSsNP in the COPD group was 51\% $(n=46)$ and over threefold higher than in the control group $(n=15, p<0.001)$ (figure 3 ). When subjects with allergic rhinitis were excluded, the diagnosis 


\begin{tabular}{|c|c|c|c|}
\hline & COPD & Control & p-value \\
\hline Subjects n & 90 & 93 & \\
\hline Males & $49(54.4)$ & 51 (54.8) & 0.96 \\
\hline Age years & $66.2 \pm 8.7$ & $63.7 \pm 8.7$ & 0.051 \\
\hline \multicolumn{4}{|l|}{ Smoking status } \\
\hline Current & $17(18.9)$ & $7(7.5)$ & $<0.001$ \\
\hline Former & $68(75.6)$ & 47 (50.5) & \\
\hline Never & $5(5.6)$ & 39 (41.9) & \\
\hline Pack-years $\#$ & $28.6 \pm 20.9$ & $6.6 \pm 10.8$ & $<0.001$ \\
\hline$B M I \mathrm{~kg} \cdot \mathrm{m}^{2}$ & $27.0 \pm 5.4$ & $27.3 \pm 4.7$ & 0.7 \\
\hline Allergic rhinitis & $5(5.6)$ & $14(15.1)$ & 0.035 \\
\hline Nasal corticosteroids & $4(4.4)$ & $4(4.3)$ & 1.0 \\
\hline \multicolumn{4}{|l|}{ Pulmonary function" } \\
\hline $\mathrm{FEV}_{1} \mathrm{~L}$ & $1.6 \pm 0.7$ & $3.0 \pm 0.9$ & $<0.001$ \\
\hline $\mathrm{FEV}_{1} \%$ pred & $53.1 \pm 18.7$ & $94.6 \pm 12.2$ & $<0.001$ \\
\hline FVC L & $3.0 \pm 1.0$ & $3.7 \pm 1.0$ & $<0.001$ \\
\hline FVC $\%$ pred & $75.8 \pm 18.0$ & $93.8 \pm 13.0$ & $<0.001$ \\
\hline $\mathrm{FEV}_{1} / \mathrm{FVC}$ & $0.53 \pm 0.12$ & $0.78 \pm 0.05$ & $<0.001$ \\
\hline \multicolumn{4}{|c|}{ 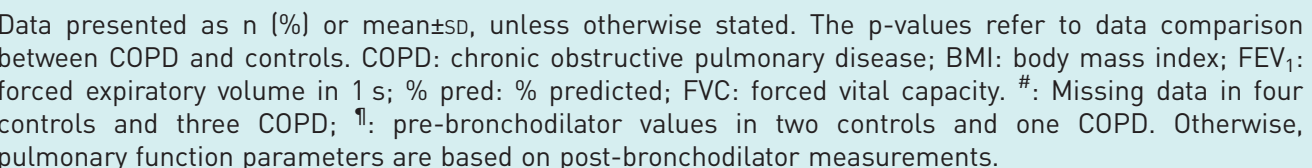 } \\
\hline
\end{tabular}

of RSsNP remained significantly greater in the COPD group than in controls (41 (48\%) versus 12 (15\%), $\mathrm{p}<0.001)$.

RSsNP was diagnosed in 47\% (32 of 68) former and 65\% (11 of 17) current smokers in the COPD group and in 19\% (9 of 47) former and 29\% (2 of 7) current smokers in the control group ( $\mathrm{p}=0.35$ ).

In the COPD group, RSsNP was diagnosed in 27 (59\%) males and $19(41 \%)$ females $(\mathrm{p}=0.41)$ and in 2 (29\%), 18 (45\%), $19(58 \%)$ and 7 (70\%) subjects with mild, moderate, severe and very severe airflow limitation (GOLD 1-4), respectively.

\section{Severity of sinonasal symptoms}

The total SNOT-22 score was significantly higher in the COPD group than in controls $(29.8 \pm 18.8$ versus $16.6 \pm 15.5, \mathrm{p}<0.001)$. SNOT subscale scores of rhinological and ear/facial symptoms were significantly higher in the COPD group than in the control group (figure $2 b$ ).

VAS for nasal obstruction, reduced sense of smell, mouth breathing, and sneezing were significantly greater in the COPD group than in the control group (table 3), while nasal discharge, facial pain and sinus pressure were not. The composite VAS score was significantly higher for rhinological symptoms in the COPD group than in the control group and was not significantly different for facial symptoms between the two groups (table 3).

\section{TABLE 2 Grade of airflow limitation in COPD subjects}

\begin{tabular}{|c|c|c|c|c|}
\hline \multirow{2}{*}{\multicolumn{2}{|c|}{ GOLD classification }} & \multicolumn{3}{|c|}{ Post-bronchodilator $\mathrm{FEV}_{1}$, mean \pm sD } \\
\hline & & \multirow{2}{*}{$\begin{array}{l}\text { n (\%) } \\
7(7.8)\end{array}$} & \multirow{2}{*}{$\begin{array}{c}\mathrm{L} \\
2.7 \pm 0.8\end{array}$} & \multirow{2}{*}{$\begin{array}{l}\% \text { pred } \\
85.7 \pm 4.2\end{array}$} \\
\hline GOLD 1 & Mild & & & \\
\hline GOLD 2 & Moderate & $40(44.4)$ & $1.9 \pm 0.6$ & $65.5 \pm 8.1$ \\
\hline GOLD 3 & Severe & $33(36.7)$ & $1.2 \pm 0.3$ & $40.0 \pm 5.3$ \\
\hline GOLD 4 & Very severe & $10(11.1)$ & $0.7 \pm 0.2$ & $24.4 \pm 3.6$ \\
\hline
\end{tabular}



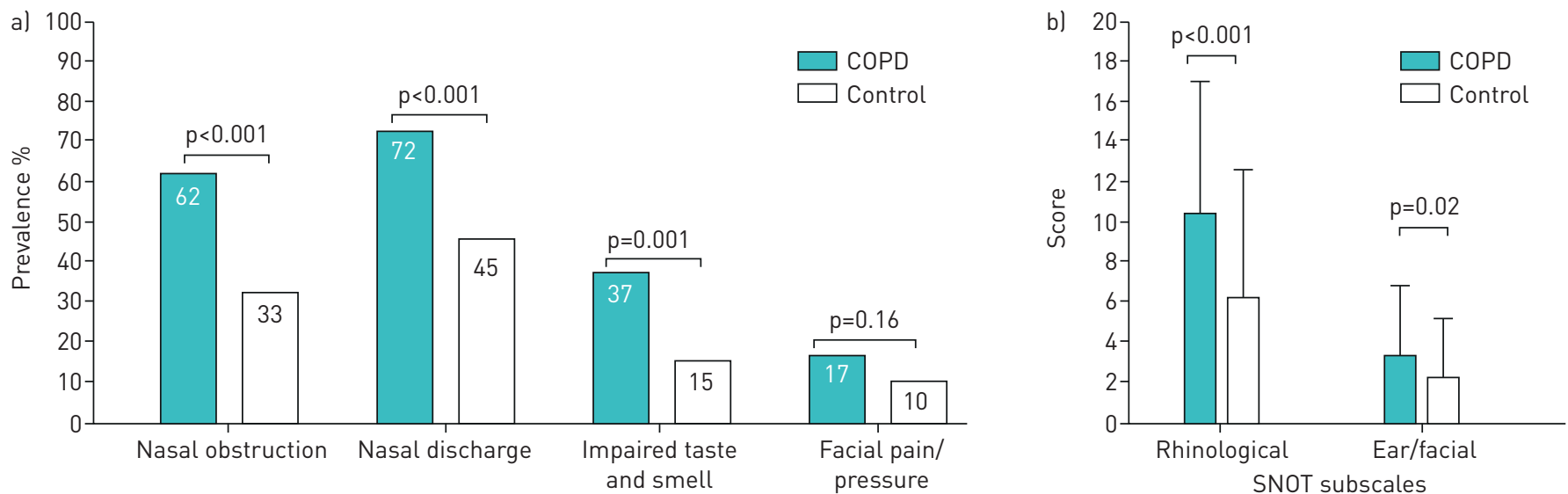

FIGURE 2 a) Prevalence of symptoms of rhinosinusitis (European position paper on rhinosinusitis and nasal polyps (EPOS) criteria). Data derived from sinonasal outcome test (SNOT)-22. b) Rhinological and ear/facial SNOT subscales. Data are presented as mean \pm SD.

Diagnostic accuracy of sinonasal symptoms for RSsNP

The area under the curve $(95 \% \mathrm{CI})$ of the ROC curve for the composite rhinological VAS was not significantly different in the COPD and control groups $(0.82(0.73-0.90)$ versus $0.85(0.76-0.95), \mathrm{p}=0.56)$. The cut-off composite rhinological VAS score was 56 and 36.5 for COPD and controls, respectively. The ROC curve values for the composite facial VAS were 0.63 (0.51-0.74) versus $0.82(0.68-0.95), p=0.03$ (figure 4).

\section{Discussion}

In this study, we found that COPD is associated with RS and sinonasal symptoms have significantly greater severity in COPD subjects than in controls. This association is supported by a clinical diagnosis of RSsNP in $51 \%$ and the presence of symptomatic criteria of RS in over $60 \%$ of COPD subjects (figure 3). Moreover, RSsNP is threefold and symptomatic and endoscopic criteria of RS are 1.5-2-fold more prevalent in the COPD group than in the control group. The greater severity of sinonasal symptoms in COPD is evident when these symptoms are assessed by SNOT-22 subscales for rhinological and ear/facial symptoms (figure $2 \mathrm{~b}$ ), VAS for individual symptoms and composite VAS for rhinological symptoms (table 3). The latter has a superior diagnostic accuracy for RS than the composite VAS for facial symptoms (figure 4).

The prevalence of nasal discharge, nasal obstruction and impaired taste and smell varied from 72 to $37 \%$ in COPD subjects in this study and was significantly greater than in controls (figure 2a). Previous studies have found that sinonasal symptoms are frequently reported by COPD patients $[10-14,25,26]$. However, those studies had limitations, such as the lack of a control group $[11,13,14,27]$ and spirometric

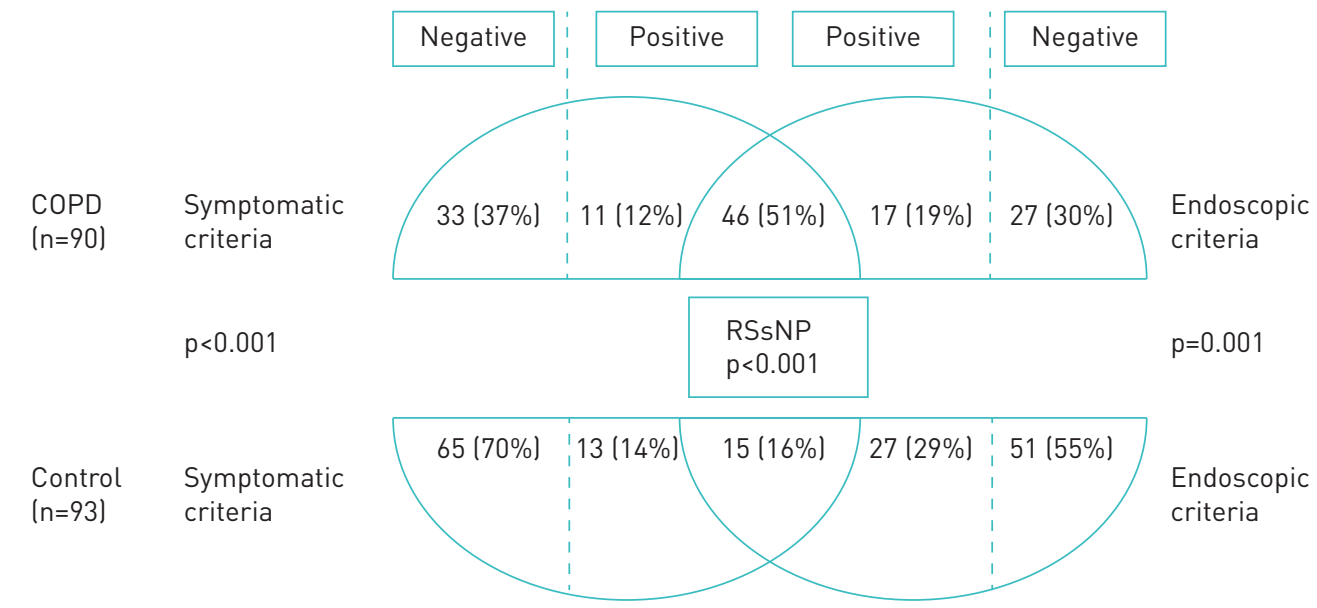

FIGURE 3 Prevalence of symptomatic and endoscopic criteria and rhinosinusitis without nasal polyps (RSsNP) in chronic obstructive pulmonary disease (COPD) and control groups. 


\section{TABLE 3 Visual analogue scales (VAS) (mm) for sinonasal symptoms}

\begin{tabular}{lccc} 
Sinonasal symptoms & COPD $(\mathbf{n = 9 0 )}$ & Control $(\mathbf{n = 9 3 )}$ & p-value \\
\hline Nasal obstruction $^{\mathbf{a}}$ & $23.4 \pm 25.9$ & $14.1 \pm 21.2$ & 0.007 \\
Nasal discharge lanterior/posterior drip) $^{\mathbf{b}}$ & $20.5 \pm 26.3$ & $13.4 \pm 21.1$ & 0.12 \\
Reduced sense of smell $^{\mathbf{c}}$ & $16.0 \pm 25.3$ & $6.9 \pm 5.4$ & 0.014 \\
Facial pain $^{\mathbf{d}}$ & $6.4 \pm 14.2$ & $4.7 \pm 12.4$ & 0.65 \\
Sinus pressure $^{\mathbf{e}}$ & $9.5 \pm 20.4$ & $6.9 \pm 16.4$ & 0.42 \\
Mouth breathing & $24.9 \pm 28.1$ & $11.9 \pm 20.1$ & 0.001 \\
Sneezing & $17.8 \pm 20.8$ & $10.8 \pm 20.1$ & 0.002 \\
Headache & $14.6 \pm 22.1$ & $10.9 \pm 19.6$ & 0.25 \\
Composite rhinological VAS (a+b+c) & $59.9 \pm 61.9$ & $34.4 \pm 51.4$ & 0.001 \\
Composite facial VAS (d+e) & $15.9 \pm 37.7$ & $11.5 \pm 27.0$ & 0.4 \\
\hline Data are presented as mean $\pm S D$, unless otherwise stated. & &
\end{tabular}

confirmation of COPD diagnosis [10, 14, 26, 27], which have been addressed in our study. Moreover, we found that almost two out of three subjects with COPD fulfilled the EPOS symptomatic criteria for RS. This prevalence is threefold higher than that reported in a Brazilian study by CAminHa et al. [21]. The difference in prevalence rates is consistent with the large variability in the overall prevalence rates of $7-27 \%$ reported across 12 countries in the 2011 pan-European prevalence study [1]. Our prevalence of $63 \%$ is slightly greater than that of $53 \%$ reported by KeLEMENCE et al. [14]. However, that study is not fully eligible for comparison, as the definition of symptomatic RS included EPOS criteria as well as headache, ear pain, halitosis and fatigue.

To our knowledge, this is the first study where EPOS endoscopic criteria were used for a diagnosis of RSsNP in COPD and control subjects. Our finding of RSsNP in every other subject with COPD is in accordance with the prevalence of $49 \%$ reported by YANG et al. [27]. However, there are differences between the two studies. In Yang's study, there was no control group; headache was included in the symptomatic criteria and radiologic criteria were used to diagnose RS. Nasal endoscopy is the only modality that discriminates RSsNP from RSwNP. Moreover, in our study, we also evaluated for allergic
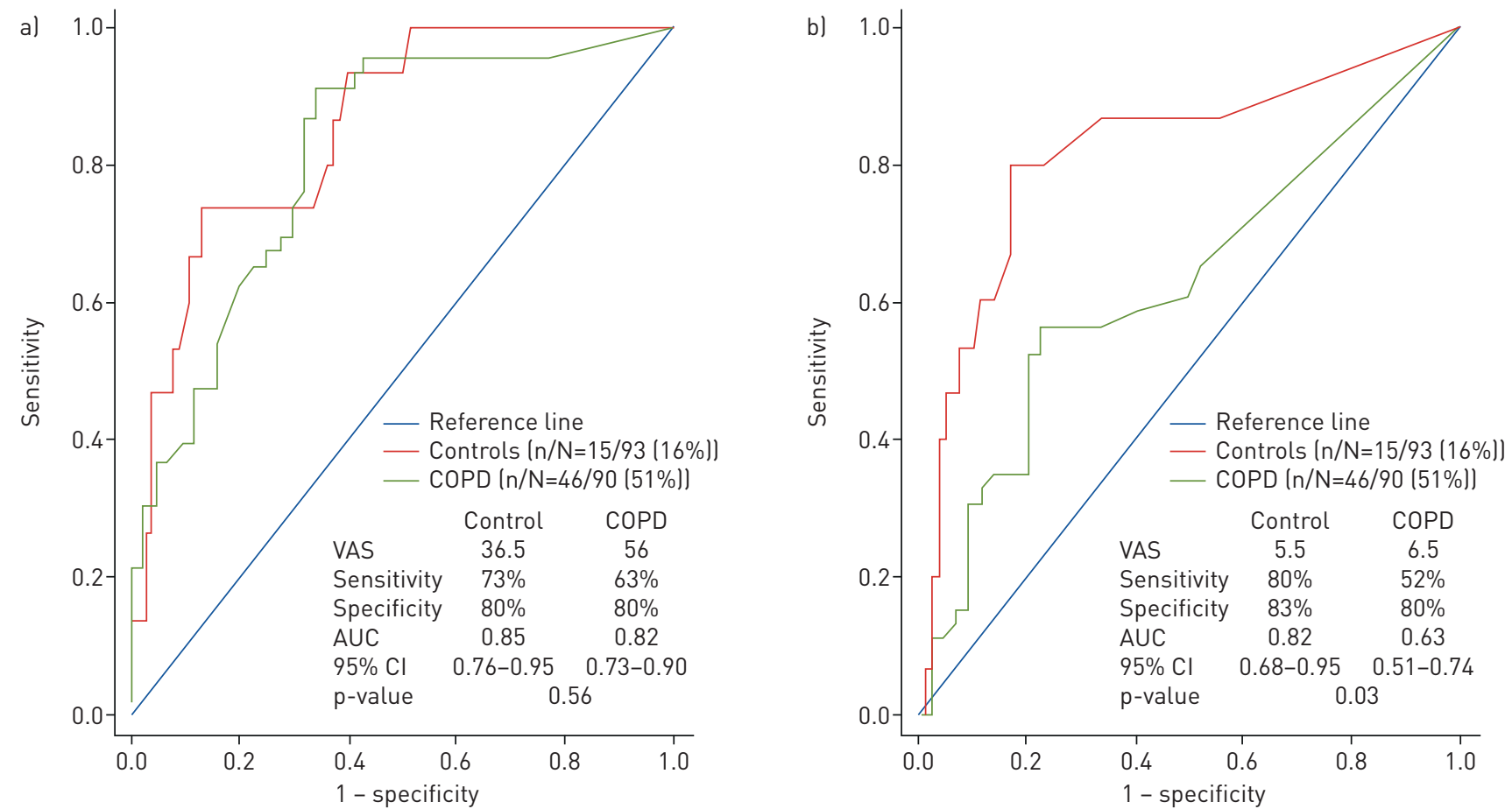

FIGURE 4 Receiver operating characteristic curves and diagnostic accuracy of (a) composite rhinological visual analogue scale (VAS) and (b) composite facial VAS in chronic obstructive pulmonary disease (COPD) and controls for a diagnosis of rhinosinusitis without nasal polyps (RSsNP) using European position paper on rhinosinusitis and nasal polyps (EPOS) criteria. AUC: area under the curve; Cl: confidence interval. 
rhinitis at the time of inclusion, as this entity affects $10-20 \%$ of the adult population [28] and can mimic symptoms of RS. Although allergic rhinitis was almost threefold more frequent in the control group, the relative prevalence of RSsNP in the COPD group was unchanged, which suggests that the high prevalence of RSsNP is not due to undiagnosed allergic rhinitis.

In addition to being more prevalent (figure 2a), the severity of RS symptoms was greater in COPD than in controls as demonstrated by data obtained from the SNOT-22 and VAS. The SNOT subscales showed that COPD subjects were significantly more troubled than controls by rhinological and ear/facial symptoms (figure $2 \mathrm{~b}$ ). This suggests that altered nasal conditions secondarily may lead to increased mouth breathing and thus inadequate protection and homeostasis of the lower airways in COPD which is in line with the UAD concept. Future studies should investigate whether RS symptoms have an impact on quality of life in COPD.

Further, the prevalence of RSsNP was $47 \%$ and 19\% in COPD and controls former smokers respectively and increased by almost 1.5 in current smokers in both groups, which may indicate that cigarette smoke has a negative effect in the upper airway. Cigarette smoke has been proposed to have pathophysiological effects on the sinonasal mucosa that can account for increased frequency of upper airway symptoms and impairment of olfaction $[29,30]$.

It would be clinically beneficial to have a simple instrument to screen COPD patients for RS. This is desirable as both patients and their physicians underrecognise sinonasal symptoms [6,31], and RS is independently associated with treatment failure in COPD exacerbations [32]. In the present study, three rhinological VAS items were summated, and the resulting composite VAS score of 56 or higher had the best diagnostic accuracy for RSsNP in COPD patients, with a sensitivity and specificity of $63 \%$ and $80 \%$, respectively. The composite VAS may have a potential for selecting COPD patients for further assessment by an otolaryngologist. However, the cut-off value for a specificity of $80 \%$ was higher in COPD than in control subjects, which may be due to differences in symptom burden in the two groups. The cut-off value may variate depending on sample characteristics and care settings [33]. As such, it will be prudent to validate the relationship between the composite rhinological VAS score and the diagnosis of RSsNP in larger cohorts of COPD patients, preferably in relation to the severity of airflow limitation, and of control subjects in primary and specialist care settings.

The strengths of this study are the relatively large sample size with almost equal sex representation, inclusion of COPD patients across the spectrum of disease severity, inclusion of a control group similar in age, number and from the same population area, as well as the use of globally accepted diagnostic criteria for RS and COPD. Spirometry with reversibility testing allowed us to eliminate asthma on COPD overlap and allergy testing permitted discrimination of RS symptoms from allergic rhinitis. There is a possibility of a recruitment bias of subjects with sinonasal symptoms in the control group, as the protocol included clinical examination by an otolaryngologist. This limitation may account for the higher mean subscale score of the SNOT rhinological symptoms in our control group, compared to the control population in a study by ERSKINE et al. [17]. Conversely, this limitation could add strength to our study as the results would be more significant if the bias was absent. Another limitation was our inability to evaluate the impact of RS on exacerbations of COPD, due to the lack of access to exacerbation data.

\section{Conclusion}

RSsNP is present in $51 \%$ of our COPD patients and is threefold more prevalent in the COPD group than in controls. These results suggest that COPD is associated with RS.

Acknowledgements: We are grateful for the assistance of the Clinical Research Facility, St. Olavs Hospital, Trondheim University Hospital and their nurses, as well as research nurse Else Bartnes, Dept of Otolaryngology, Head and Neck Surgery, St. Olavs University Hospital.

Conflict of interest: None declared.

Support statement: The study was supported by a $\mathrm{PhD}$ grant provided by the Liaison Committee between the Central Norway Regional Health Authority and the Norwegian University of Science and Technology.

\section{References}

1 Hastan D, Fokkens WJ, Bachert C, et al. Chronic rhinosinusitis in Europe-an underestimated disease. A GA(2) LEN study. Allergy 2011; 66: 1216-1223.

2 Fokkens WJ, Lund VJ, Mullol J, et al. European position paper on rhinosinusitis and nasal polyps 2012. Rhinol Suppl 2012; 23: 1-298.

3 Bachert C, Pawankar R, Zhang L, et al. ICON: chronic rhinosinusitis. World Allergy Organ J 2014; 7: 25.

4 Ahn JC, Kim JW, Lee CH, et al. Prevalence and risk factors of chronic rhinosinusitis, allergic rhinitis, and nasal septal deviation: results of the Korean national health and nutrition survey 2008-2012. JAMA Otolaryngol Head Neck Surg 2016; 142: 162-167. 
Passalacqua G, Ciprandi G, Canonica GW. United airways disease: therapeutic aspects. Thorax 2000; 55: S26-S27.

6 Hox V, Maes T, Huvenne W, et al. A chest physician's guide to mechanisms of sinonasal disease. Thorax 2015; 70: 353-358.

7 Feng $\mathrm{CH}$, Miller MD, Simon RA. The united allergic airway: connections between allergic rhinitis, asthma, and chronic sinusitis. Am J Rhinol Allergy 2012; 26: 187-190.

8 Global Initiative for Chronic Obstructive Lung Disease. Global strategy for the diagnosis, management, and prevention of chronic obstructive pulmonary disease 2020 report. https:/goldcopd.org/wp-content/uploads/2019/ 12/GOLD-2020-FINAL-ver1.2-03Dec19_WMV.pdf

9 Barnes PJ. Inflammatory endotypes in COPD. Allergy 2019; 74: 1249-1256.

10 Caillaud D, Chanez P, Escamilla R, et al. Association of chronic nasal symptoms with dyspnoea and quality-of-life impairment in chronic obstructive pulmonary disease. Respirology 2014; 19: 346-352.

11 Hurst JR, Wilkinson TM, Donaldson GC, et al. Upper airway symptoms and quality of life in chronic obstructive pulmonary disease (COPD). Respir Med 2004; 98: 767-770.

12 Montnemery P, Svensson C, Adelroth E, et al. Prevalence of nasal symptoms and their relation to self-reported asthma and chronic bronchitis/emphysema. Eur Respir J 2001; 17: 596-603.

13 Roberts NJ, Lloyd-Owen SJ, Rapado F, et al. Relationship between chronic nasal and respiratory symptoms in patients with COPD. Respir Med 2003; 97: 909-914.

14 Kelemence A, Abadoglu O, Gumus C, et al. The frequency of chronic rhinosinusitis/nasal polyp in COPD and its effect on the severity of COPD. COPD 2011; 8: 8-12.

15 Hakansson K, Konge L, Thomsen SF, et al. Sinonasal inflammation in COPD: a systematic review. Eur Respir J 2013; 42: 1402-1411.

16 Lange B, Thilsing T, Al-kalemji A, et al. The sinonasal outcome test 22 validated for Danish patients. Dan Med Bull 2011; 58: A4235.

17 Erskine SE, Hopkins C, Clark A, et al. SNOT-22 in a control population. Clin Otolaryngol 2017; 42: 81-85.

18 Miller MR, Hankinson J, Brusasco V, et al. Standardisation of spirometry. Eur Respir J 2005; 26: 319-338.

19 Crapo RO, Morris AH, Gardner RM. Reference spirometric values using techniques and equipment that meet ATS recommendations. Am Rev Respir Dis 1981; 123: 659-664.

20 Heinzerling L, Mari A, Bergmann KC, et al. The skin prick test - European standards. Clin Transl Allergy 2013 ; 3: 3.

21 Caminha GP, Pizzichini E, Lubianca Neto JF, et al. Rhinosinusitis symptoms, smoking and COPD: prevalence and associations. Clin Otolaryngol 2018; 43: 1560-1565.

22 Bousquet PJ, Combescure C, Neukirch F, et al. Visual analog scales can assess the severity of rhinitis graded according to ARIA guidelines. Allergy 2007; 62: 367-372.

23 Lim M, Lew-Gor S, Darby Y, et al. The relationship between subjective assessment instruments in chronic rhinosinusitis. Rhinology 2007; 45: 144-147.

24 Mandrekar JN. Receiver operating characteristic curve in diagnostic test assessment. J Thorac Oncol 2010; 5: $1315-1316$.

25 Hens G, Vanaudenaerde BM, Bullens DM, et al. Sinonasal pathology in nonallergic asthma and COPD: 'united airway disease' beyond the scope of allergy. Allergy 2008; 63: 261-267.

26 Piotrowska VM, Piotrowski WJ, Kurmanowska Z, et al. Rhinosinusitis in COPD: symptoms, mucosal changes, nasal lavage cells and eicosanoids. Int J Chron Obstruct Pulmon Dis 2010; 5: 107-117.

27 Yang $\mathrm{X}, \mathrm{Xu} \mathrm{Y}$, Jin J, et al. Chronic rhinosinusitis is associated with higher prevalence and severity of bronchiectasis in patients with COPD. Int J Chron Obstruct Pulmon Dis 2017; 12: 655-662.

28 Brozek JL, Bousquet J, Baena-Cagnani CE, et al. Allergic rhinitis and its impact on asthma (ARIA) guidelines: 2010 revision. J Allergy Clin Immunol 2010; 126: 466-476.

29 Reh DD, Higgins TS, Smith TL. Impact of tobacco smoke on chronic rhinosinusitis: a review of the literature. Int Forum Allergy Rhinol 2012; 2: 362-369.

30 Kjaergaard T, Cvancarova M, Steinsvaag SK. Smoker's nose: structural and functional characteristics. Laryngoscope 2010; 120: 1475-1480.

31 Milkowska-Dymanowska J, Bialas AJ, Zalewska-Janowska A, et al. Underrecognized comorbidities of chronic obstructive pulmonary disease. Int J Chron Obstruct Pulmon Dis 2015; 10: 1331-1341.

32 Dewan NA, Rafique S, Kanwar B, et al. Acute exacerbation of COPD: factors associated with poor treatment outcome. Chest 2000; 117: 662-671.

33 Soreide K, Korner H, Soreide JA. Diagnostic accuracy and receiver-operating characteristics curve analysis in surgical research and decision making. Ann Surg 2011; 253: 27-34. 